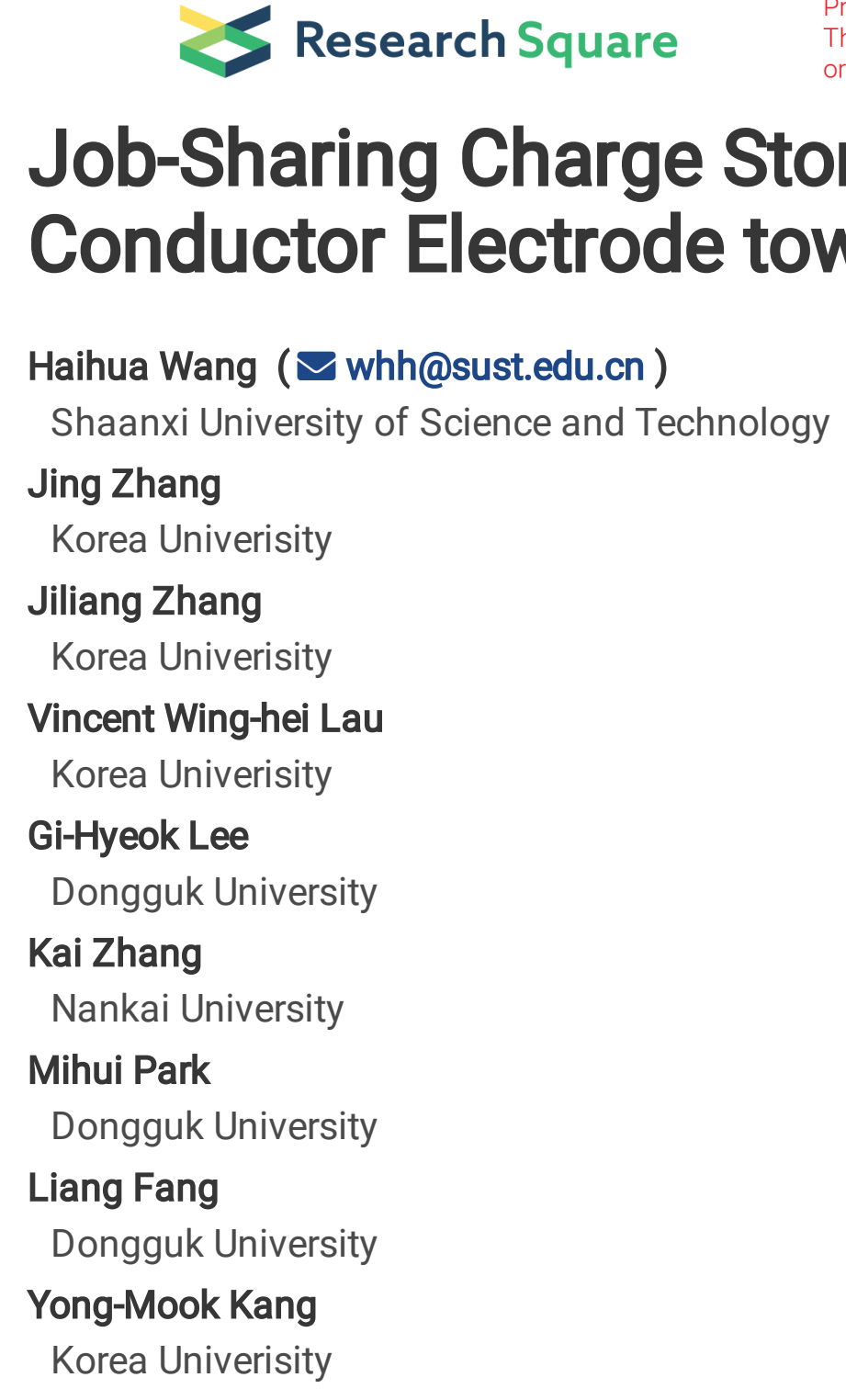

\author{
Haihua Wang ( $\nabla$ whh@sust.edu.cn ) \\ Shaanxi University of Science and Technology \\ Jing Zhang \\ Korea Univerisity \\ Jiliang Zhang \\ Korea Univerisity \\ Vincent Wing-hei Lau \\ Korea Univerisity \\ Gi-Hyeok Lee \\ Dongguk University \\ Kai Zhang \\ Nankai University \\ Mihui Park \\ Dongguk University \\ Liang Fang \\ Dongguk University \\ Yong-Mook Kang \\ Korea Univerisity
}

\title{
Job-Sharing Charge Storage in a Mixed Ion/Electron Conductor Electrode towards Ultrafast Na Storage
}

\section{Physical Sciences - Article}

Keywords: Prussian Blue, Fast kinetics, Job-sharing charge storage, Interfacial storage, Na rechargeable batteries

Posted Date: November 30th, 2020

DOI: https://doi.org/10.21203/rs.3.rs-94792/v2

License: (c) (1) This work is licensed under a Creative Commons Attribution 4.0 International License. Read Full License 


\section{Job-Sharing Charge Storage in a Mixed Ion/Electron Conductor Electrode towards}

2 Ultrafast Na Storage

3 Jing Zhang ${ }^{1}$, Jiliang Zhang ${ }^{1}$, Vincent Wing-hei Lau ${ }^{1}$, Haihua Wang ${ }^{2 *}$, Gi-Hyeok Lee ${ }^{3}$, Kai Zhang ${ }^{4}$,

4 Mihui Park ${ }^{3}$, Liang Fang ${ }^{3}$, and Yong-Mook Kang ${ }^{1 *}$

$5 \quad{ }^{1}$ Department of Materials Science and Engineering, Korea University, Seoul 02841, Republic of Korea

$6 \quad{ }^{2}$ College of Chemistry and Chemical Engineering, Shaanxi University of Science and Technology, Xi'an, $7 \quad$ 710021, PR China

$8 \quad{ }^{3}$ Department of Energy and Materials Engineering, Dongguk University-Seoul, Seoul 04620, Republic of 9 Korea

$10{ }^{4}$ Key Laboratory of Advanced Energy Materials Chemistry (Ministry of Education), Renewable Energy 11 Conversion and Storage Center, College of Chemistry, Nankai University, Tianjin 300071, China

12 E-mail: dake1234@korea.ac.kr (Y.-M. Kang),whh@sust.edu.cn (H. Wang)

13 KEYWORDS: Prussian Blue, Fast kinetics, Job-sharing charge storage, Interfacial storage, Na 14 rechargeable batteries

For the cathode materials potentially available for high power capability, reducing their particle size can

17 improve the bulk ionic conductivity due to reduced ion diffusion length, and exploiting new reaction mechanism must be fundamentally advantageous. However, other issues such as synthesis difficulty, poor charge storage stability, and capacity decay can emerge. To simultaneously address these issues, in this work, we first find solid-solid interfacial storage for the ultrafine insertion cathode materials in the spacecharge region of a mixed ion/electron conductor through the so-called "job-sharing" mechanism. This 
mechanism shows that electrons and ions can be stored in the different phases around the interface and transport only inside there, which looks thermodynamically distinct from most of conventional charge storage mechanisms in terms of the relationship between charge storage and cell voltage. The insertion cathodes governed by the "job-sharing" mechanism thus exhibit the outstanding performances with high capacity, fast kinetics, and stable cyclability. Herein, the inverse conceptual compositing between ionic conductor and electronic conductor to harness the size effect offers a potential research direction for not only electrode design in high-power batteries, but also other electrochemical potential applications such as solid-state electrolytes and so on.

Reversible electrochemical systems integrating high energy secondary battery and high power capacitor look ideal, as they can simultaneously meet various demands of mobile electronic devices toward the use for a longer time as well as electric vehicles toward a wider driving range and higher power capability. The demand must be more challenging for sodium ion batteries (SIBs) due to the bigger size of $\mathrm{Na}^{+}$ion (102 pm) than that of $\mathrm{Li}^{+}$ion $(76 \mathrm{pm})$. A simple and straightforward approach to render secondary batteries to be governed by faster kinetics for higher power capability is to reduce the particle size of electrode materials to minimize the distance for ion transfer. ${ }^{1}$ Particle miniaturization offers a high surface-tovolume ratio, thereby exposing more surface sites as opposed to bulk ones. The surface atoms generally have higher Gibbs free energy and more plentiful defects than its bulk homologue. Hence, the size confinement effect in electrode materials is not limited to shortened ion transfer pathways but can also induce unexpectedly rapid movement of electrons and ions in the enlarged space charge region which may stem from higher contents of surface atoms. ${ }^{2}$ Many unique physicochemical characteristics are manifested in size-confined electrode materials, such as the variation of lattice parameter(s), ${ }^{3-5}$ chemical potential, ${ }^{6,7}$ and electronic structure ${ }^{8}$ all of which can affect their electrochemical behaviors. As for the 
cathode materials, downsizing is reported to narrow the miscibility gap for phase transitions, facilitating solid solution reactions for improved cyclic stability and reaction kinetics. ${ }^{9}$ Moreover, the solid solution reaction facilitated by the size confinement can be typically found in some cathode materials like $\mathrm{LiFePO}_{4}$ and $\mathrm{LiMn}_{2} \mathrm{O}_{4}$ because of the enhanced Li solubility in their nanocrystalline homologues. ${ }^{10,11}$ However, the downsizing strategy is rarely reported for cathode materials, and in many cases, the size confinement with cathode materials often results in the significant decrease of their capacities due to the breakage of internal insertion sites in their crystal structures and the following reduction of inserted ions. ${ }^{3}$ Hence, accentuating the merits of size confinement with cathode materials and mitigating its demerits are worth being explored for innovating the cathode materials for alkali ion secondary batteries.

Novel mechanism for cathode materials should be developed and exploited to innovate the state-of-theart battery technologies. Adjusting the crystal structures of cathode materials can modulate the reaction mechanism toward alkali ions, resultantly improving their energy densities, reversibility, and kinetics. ${ }^{12-}$ ${ }^{15}$ The introduction of reversible $\mathrm{Mn}(\mathrm{II}) / \mathrm{Mn}(\mathrm{IV})$ redox couple can greatly increase the capacity of lithiumexcess Mn-based cathode. ${ }^{13}$ Putting hetero-species like crystal water and so on into a layered structure (birnessite) cathode exploited the unprecedentedly reversible phase transition between the layered and a metastable spinel-like structure for higher capacity as well as better cyclic stability. ${ }^{14}$ However, the number of cathode materials governed by these new mechanisms is few, and so the brand-new mechanism which can be generally applied for a lot of cathode materials is urgently required. Recently, a new interfacial storage mechanism named job-sharing storage which differs from conventional reaction mechanisms like intercalation, conversion, etc. has been proposed by Maier et al. ${ }^{7,16-19}$ This mechanism reveals that positive and negative charges can be stored separately in two different phases within nanocomposites, contributing additional capacity independent of bulk storage. The reaction kinetics could be ultrafast because the transfer of two charge carriers is also independent. The job-sharing storage was 
reported to primarily govern the reaction kinetics of transition metal oxides which depend on conversion reaction. However, this mechanism has never been demonstrated in the electrode materials with other reaction mechanisms such as intercalation and so on. Because the job-sharing storage is limited in the space-charge region and thus the following capacity and kinetics are determined by the ratio of boundary region, its universal extension to intercalation and so forth could be accelerated by our size confinement strategy with cathode materials.

Herein, for the first time, we report the application of this brand-new job-sharing mechanism for sizeconfined insertion-type cathode materials to achieve a high energy, high power, and highly stable battery. We selected Prussian blue analogues (PBAs), one of the commonly used cathodes for SIBs, as a proof of concept to fabricate an artificial mixed conductor by compositing iron hexacyanoferrate nanodots (FeHCF NDs) as an ionic conductor with reduced graphene oxide (rGO) as an electron conductor. This composite was synthesized by a two-step ion-exchange method with FeOOH NDs/rGO composite as a precursor (Fig. 1a, Supplementary Fig. 1-3). The strong interaction between FeHCF NDs and rGO enables the ion and electron pathway to be decoupled within the space-charge region of this composite. The job-sharing mechanism was well clarified, which is totally different from the traditional charge storage mechanisms such as intercalation, conversion and so on. The capacity-voltage relation of interfacial storage in the three modes is updated against that of the bulk one. The FeHCF NDs/rGO composite thus shows beneficial interface/surface behavior and excellent electrochemical performance. Therefore, the mixed electron/ion conductor, FeHCF NDs/rGO, paves a novel way for the charge storage for alkali ion secondary batteries.

\section{Material characterizations}

Field emission scanning electron microscopy (FESEM), transmission electron microscope (TEM), and high-resolution TEM (HRTEM) images of FeHCF NDs/rGO (Fig. 1b-e, Supplementary Fig. 4) show that the ultrafine nanodots are tightly and uniformly embedded into the graphene sheets with a monodisperse 
91 feature and a homogeneous distribution of $\mathrm{Na}, \mathrm{Fe}, \mathrm{C}$, and $\mathrm{N}$ (Supplementary Fig. 5). The lattice fringes 92 with a d-spacing of $0.258 \mathrm{~nm}$ correspond to the (400) plane of FeHCF. The nanodots have an average size of $9.7 \mathrm{~nm}$ (Fig. 1f), which is one of the smallest particle sizes of high-quality PBAs in the consensus reports. For comparison, a poorly contacted FeHCF microcubes/rGO composite (FeHCF MCs/rGO; Supplementary Fig. 6) with particle size up to submicron $(\sim 293 \mathrm{~nm})$ was formed when higher ascorbic acid amount (50 mg) was used ( $2 \mathrm{mg}$ for FeHCF NDs/rGO), resulting in a much lower specific surface area $\left(57.6 \mathrm{~m}^{2} \mathrm{~g}^{-1}\right)$ than FeHCF NDs/rGO $\left(148 \mathrm{~m}^{2} \mathrm{~g}^{-1}\right)$ (Supplementary Fig. 7$)$.

The formula (Supplementary Fig. 8, Supplementary Table 1) of FeHCF MCs/rGO and FeHCF $\mathrm{NDs} / \mathrm{rGO}$ are quantified to be $\mathrm{Na} 0.89 \mathrm{FeFe}(\mathrm{CN})_{6} \cdot 1.86 \mathrm{H}_{2} \mathrm{O}$ and $\mathrm{Na}_{0.96} \mathrm{FeFe}(\mathrm{CN})_{6} \cdot 2.09 \mathrm{H}_{2} \mathrm{O}$, respectively. The powder X-ray diffraction (XRD) patterns (Fig. 2a) of the two samples are indexed to the facepeaks of FeHCF NDs/rGO are attributed to its small size. The calculated lattice parameter of FeHCF $\mathrm{NDs} / \mathrm{rGO}(a=10.255 \AA)$ is larger than that of FeHCF MCs $/ \mathrm{rGO}\left(a_{0}=10.221 \AA\right)$, revealing the lattice expansion of ultrafine crystals (Supplementary Table 2, Supplementary Note 1). The size-dependent lattice expansion has been found in a lot of materials, such as $\mathrm{LiCoO}_{2}, \mathrm{CeO}_{2}, \mathrm{TiO}_{2}$ (rutile), $\mathrm{BaTiO}_{3}$, $\mathrm{MnCr}_{2} \mathrm{O}_{4}, \mathrm{ZnO}, \mathrm{Ni}$, and $\mathrm{MgO}^{3,22-24}$ The different features of Fe K-edge X-ray absorption near-edge structure (XANES) spectra (Fig. 2b, Supplementary Note 2) for the two samples are attributed to the high surface atom ratio and the low crystallinity of size-confined sample that cause the different types of ligand bonded with the $\mathrm{Fe}$ atoms at the surface. The poor symmetry of the related extended X-ray absorption fine structure (EXAFS) spectrum (Fig. 2c) reveals the distortion of the structure for FeHCF ND compared with FeHCF MCs. ${ }^{25}$ 
The Raman spectrum (Fig. 2d, Supplementary Note 3) of FeHCF NDs/rGO shows a significant 113 reduction of intensity for the $\mathrm{C} \equiv \mathrm{N}$ vibration compared with that of $\mathrm{FeHCF} \mathrm{MCs} / \mathrm{rGO}$, suggesting that the 114 shrinkage of the long-range structure suppresses the vibrational stretching mode of the ultrafine 115 particles. ${ }^{26}$ Besides, size-induced frequency shift to lower wavenumber is observed (Supplementary Table 116 2), which is partially caused by the increase of bond distance resulting from lattice expansion and may 117 partly be due to the phonon confinement effect within the small sized grains. ${ }^{3,27}$ A peak shift to lower 118 binding energy is observed in the Fe 2p X-ray photoelectron spectroscopy (XPS) spectra (Fig. 2e, 119 Supplementary Table 2, Supplementary Note 3) of FeHCF NDs/rGO, revealing Fe in Prussian blue 120 attracts electrons from the $\pi-\pi$ conjugated system of graphene. ${ }^{28}$ It further indicates the interaction within 121 the close contact interface of the composite. Similar conclusion can be drawn from N 1s spectra (Fig. 2f) 122 that show an increase in relative peak intensities of $\mathrm{N}(399.8 \mathrm{eV})$ and $\mathrm{N}(402.4 \mathrm{eV})$ to $\mathrm{N}(397.7 \mathrm{eV})$ for 123 FeHCF NDs/rGO. In short, the ultrafine FeHCF nanocrystallines show expanded lattice, low degree of 124 order, high structural distortion, and intense interaction with graphene layers.

\section{Electrochemical behavior}

126 Both the cyclic voltammograms (CV) and galvanostatic charge/discharge profiles (Fig. 3a, c) of FeHCF $127 \mathrm{MCs} / \mathrm{rGO}$ have the typical profile of bulk sodium iron hexacyanoferrates with two redox couples 128 (Supplementary Note 4). After downsizing, they show a different characteristic with weakened peaks and 129 platforms (Fig. 3b, d). Both of the curves show enhanced capacity in the lower voltage region. The 130 contraction of the miscibility gap that makes the charge/discharge curves of FeHCF NDs/rGO smoother 131 (Fig. 3d) suggests that the thermodynamics and kinetics associated with phase-transition in size-confined 132 particles are different from those in bulk-sized particles. ${ }^{29}$ This phenomenon appears in many size 133 confinement systems..$^{3,9,10,30}$ 
Now we take the mixed conductor into account and focus on the interface between FeHCF and rGO in

the space-charge zone. Electroneutrality is governed by Poisson-Boltzmann's equation which depends on the difference in structure and electrical potential at the interface and in the bulk. ${ }^{17}$ As a result, the charge carriers (including electron and ion) are re-distributed and can be considered independently at the interface (but are coupled in the bulk). ${ }^{31}$ A contour map (Fig. 3e) of charge carrier concentration derived from Gouy-Chapman profiles shows the redistribution of charge carriers at the FeHCF NDs/rGO interface, indicating mutative charge storage from bulk to interface. In this case, the charge storage in the interface complies with a job-sharing mechanism illustrated in Fig. 3f, which suggests that the ions (e.g., $\mathrm{Na}^{+}$) can store and transport in one phase (e.g., PB) and electrons in the other phase (e.g., graphene) within the twophase interface. This storage is heavily dependent on size effect and two-phase contacts. The relationship between charge $Q$ stored in the space-charge zone and the particle thickness $l$ (Supplementary Fig. 9) illustrates how $Q$ increases in nanoscale materials and even more so for atomic-scale size. ${ }^{7}$ In our case, the space-charge zone covers a large part of the FeHCF ND particle. Both the contact area and spacecharge zone of FeHCF NDs/rGO are much larger than those of FeHCF MCs/rGO. Therefore, interfacial storage contributes or even dominates the mass storage mechanism for the ND composite, but is negligible for FeHCF MCs/rGO. Experimental verification (Supplementary Fig. 10) suggests that the capacity of the composite is not just provided by the single phase FeHCF NDs or rGO; more importantly, the interfacial storage also makes a great contribution to the total capacity of the composite.

For simplicity (ignoring intrinsic contribution), the thermodynamic description of the relationship between $\mathrm{Na}$ activity $a_{\mathrm{Na}}$ and job-sharing stored charge $Q$ is given via $a_{N a} \propto Q^{n} \exp (k Q)$ (calculation cf. ref. $^{17}$ ), where $n$ is between 3 and 4 , depending on the condition of contact phases, and $k$ is a constant. Note that this equation represents the capacity-voltage relation according to $E=$ const. $-(R T / F) \ln a_{\mathrm{Na}}$. In the bulk, however, the relation follows $a_{\mathrm{Na}} \propto Q^{2}$. The differences are shown in the illustration of the $\ln a_{\mathrm{Na}}-$ 
$157 Q$ profiles (Supplementary Fig. 11). The job-sharing storage contains three modes as given by (for more 158 details, see Methods): (i) intrinsic characteristics ( $a_{\mathrm{Na}}=$ const.); (ii) diffusive-layer ( $\left.a_{\mathrm{Na}} \propto Q^{n}\right)$; and (iii) 159 rigid-layer $\left(a_{\mathrm{Na}} \propto \exp (k Q)\right)$. To reveal the correlation, we fitted $\ln Q v s . E$ plots (Fig. $\left.3 \mathrm{~g}\right)$ derived from 160 the discharge curve of FeHCF NDs/rGO at different voltages (Supplementary Fig. 12). The power-law 161 exponent $n$ only at a high voltage range of 3.5-3.9 $\mathrm{V}$ is between 3 and 4 , indicating the charge storage 162 dominated by a diffuse layer. The power-law function demonstrates an irrational fit $(n<3)$ when voltage 163 is lower than $3.5 \mathrm{~V}$. To reveal the effect of a rigid layer, we plotted $E+n R T \ln Q / F v s . Q$ with $n=3$ and 4 , 164 respectively (Fig. 3h). ${ }^{32}$ The plots reveal a linear correlation when $Q>60 \mathrm{mAh} \mathrm{g}^{-1}$ with a $k$ value of 0.01 165 for both $n=3$ and 4 . The job-sharing storage is overall based on the rigid-layer mode that is applicable in a wide voltage range, consistent with extra capacity observed in the CV and charge/discharge curves. In this regime, the storage shows a capacitor-like behavior that functions in the solid/solid interface.

One advantage of the job-sharing effect is to provide extra capacity. The insertion capacity of ion insertion type framework is decreasing with the reduction of particle size. The insertion capacity of FeHCF loses nearly $20 \mathrm{mAh} \mathrm{g}^{-1}$ from bulk to a $10 \mathrm{~nm} \times 10 \mathrm{~nm} \times 10 \mathrm{~nm}$ cube (Methods, Supplementary Fig. 13, Fig. 3i). However, the two samples show the same capacity at $0.2 \mathrm{C}$, which is credited to the high interface storage as mentioned above.

\section{Electrochemical reaction kinetics}

Mass transfer in the mixed ion/electron conductor could be very fast due to the size confinement effect and the job-sharing mechanism. This is confirmed by the superior rate ability (Fig. 4a) of FeHCF $\mathrm{NDs} / \mathrm{rGO}$. The charge-discharge curves of FeHCF NDs/rGO (Fig. 4b) at various current densities (0.2 C to $50 \mathrm{C}$ ) display similar curve shape, while those of FeHCF MCs/rGO (Fig. 4c) appear to have obvious polarization at high C-rates. Furthermore, FeHCF MCs/rGO shows higher overpotential at high rates, 
179 leading to a higher voltage hysteresis (Supplementary Fig. 14), which are indicative of reduced kinetics 180 and energy efficiency.$^{33}$ For comparison, the rate capability of FeHCF NDs/rGO (Fig. 4d) is superior to 181 other state-of-the-art PBA-based cathodes, ${ }^{25,34-41}$ especially at high current densities.

To account for the superior rate capability of FeHCF NDs/rGO, we employed several methodologies to investigate the origin of the outstanding electrochemical reaction kinetics. The difference of the calculated $b$-values (Methods, Supplementary Fig. 15a, b, Fig. 4e) indicates both diffusion-controlled and capacitive processes in the Na storage mechanism of FeHCF NDs/rGO, of which the latter dominates,$^{30}$ while Na storage for FeHCF MCs/rGO is largely diffusion-controlled at a low scan rate but is more capacitive with increasing scan rate. Note that this capacitive behavior includes common pseudocapacitance and interfacial storage, and both of them result in fast charge/discharge. However, according to previous publications reporting that size reduction of the cathode material in and of itself leads to lower capacity, ${ }^{3,42}$ we believe the extra capacity in FeHCF NDs/rGO is mostly contributed by interfacial storage. The calculated surface/interface contribution ratios (Methods, Fig. 4f, Supplementary Fig. 15c) of the two electrodes show the same conclusion as the $b$-value. Note that, at a high rate, diffusivity is the bottleneck to capacity contribution of FeHCF MCs/rGO. jointly are factors to ultra-fast kinetics. Generally speaking, ion diffusion is the rate-determining step for the electrochemical reaction. The diffusion time $\left(\tau^{\delta}\right)$ is proportional to $L^{2} / D^{\delta}$, where $L$ is the diffusion length and $D^{\delta}$ is the diffusion coefficient, ${ }^{43}$ so shortening $L$ or increasing $D^{\delta}$ can promote the diffusion kinetics. In our case, the $L$ for FeHCF MCs is almost 30 times larger than that of FeHCF NDs on average, meaning that the diffusion time of the former is almost 900 times higher than that of the latter, assuming an identical $D^{\delta}$ in both cases. On the other hand, diffusion time $\tau^{\delta}$ also refers to transport resistance $\mathrm{R}^{\delta}$ and 
$1 / \mathrm{C}^{\delta}$, when $L$ is fixed. $D^{\delta}$ is expressed differently for bulks and interfaces (Supplementary Table 3). By 203 inspecting these expressions, two aspects are evident that can rationalize why $D_{\text {interface }}$ is greater than $D_{\text {bulk. }}{ }^{18,19}$ (i) In bulk materials, the ions and electrons are transferred along the same pathway, whereas in the job-sharing interface, they are decoupled and are transported within their respective conductor (Fig. 3f), leading to a low resistance $\mathrm{R}^{\delta}$. (ii) For the job-sharing mode, there is an extra term $\left(\frac{F^{2} s^{2}}{R T \varepsilon \varepsilon_{0}}\right)$ related to the electrostatic energy in $1 / \mathrm{C}^{\delta}$ that reduces the capacitance term $\mathrm{C}^{\delta}$.

The Na ion diffusion coefficient $D_{\mathrm{Na}}\left(\mathrm{cm}^{2} \mathrm{~s}^{-1}\right)$ can be calculated via the galvanostatic intermittent titration technique (GITT) (Methods, Supplementary Fig. 16, Fig. 4g). ${ }^{44,45}$ FeHCF NDs/rGO overall shows a higher $D_{\mathrm{Na}}$ value $\left(10^{-8}\right.$ to $\left.10^{-11} \mathrm{~cm}^{2} \mathrm{~s}^{-1}\right)$ compared with FeHCF MCs $/ \mathrm{rGO}\left(10^{-10}\right.$ to $\left.10^{-13} \mathrm{~cm}^{2} \mathrm{~s}^{-1}\right)$ over both charging and discharging, and is higher than those of many other PBA-based cathodes as well. ${ }^{40,46}$ To sum up, the combination of the small diffusion length and the high diffusion coefficient lead to the fast ion diffusion kinetics of FeHCF NDs/rGO electrode, which removes the solid-state diffusion as a limiting factor and makes the surface/interface storage the dominant contributor to capacity. For 215 FeHCF MCs/rGO, however, the longer diffusion path and the low diffusion coefficient for Na ions induce a diffusion-controlled process at low C-rate. At high current density, which requires short reaction time, Na ions probably only diffuse over a short-distance and occupy near-surface reaction sites within FeHCF MCs/rGO (Supplementary Fig. 17). Ex-situ XPS analyses (Supplementary Fig. 18, Fig. 4h, Supplementary Table 4) confirm that Na ions are only inserted into the near-surface sites of FeHCF MCs at high $\mathrm{C}$-rate. Besides, a lower charge transfer resistance $\left(\mathrm{R}_{\mathrm{ct}}\right)$ and a higher diffusion coefficient obtained

221 from electrochemical impedance spectroscopy (EIS) (Supplementary Fig. 19) confirm the superiority of 222 the size-confined sample further. 
Fig. 4i shows schematic graphical summary of the kinetic mechanism where the interface, surface, and crystal interior act in concert for charge storage. The extensive contact of the two conductors and highly exposed surfaces contribute to high interfacial (solid-solid contact) and surface (solid-electrolyte contact) storage. This charge storage mechanism shows an excellent kinetic characteristic. The job-sharing transport ensures the high conductivity of both electrons and ions that allow fast $\mathrm{Na}^{+}$diffusion in the internal structure owing to the much more shortened ion diffusion length.

\section{Electrochemical reaction stability}

Fig. 5a shows the capacity retention upon cycling for the two electrodes. An outstanding cyclability of FeHCF NDs/rGO is observed, showing a capacity of $128 \mathrm{mAh} \mathrm{g}^{-1}$ over 100 cycles with only $3 \%$ capacity loss. However, for FeHCF MCs/rGO, the capacity decreases to $102 \mathrm{mAh} \mathrm{g}^{-1}$ after 100 cycles with a capacity retention of $77.9 \%$. The high stability of FeHCF NDs/rGO is also confirmed by the stable

Coulombic efficiency (CE), the value of which stabilizes to $99.5+\%$ after 8 cycles. In contrast, the CE of

FeHCF MCs/rGO is more erratic after 26 cycles, and swings between $97.5 \%$ and $101.4 \%$. The superb structural stability of the FeHCF NDs/rGO was further verified by cycling at $10 \mathrm{C}$ (Fig. 5b), showing a high capacity changing from $104 \mathrm{mAh} \mathrm{g}^{-1}$ to $84 \mathrm{mAh} \mathrm{g}^{-1}$ over 1000 cycles with a high and stable CE of 99+\%. The full cell test (Supplementary Fig. 20, 21) with a good electrochemical performance suggests the practical applicability of FeHCF NDs/rGO sample for high-energy and high-power batteries. 5). The structures of both electrodes (Fig. 5d, h) reversibly evolve between rhombohedral (R) phase $\left(\mathrm{Na}_{2} \mathrm{Fe}^{\mathrm{II}} \mathrm{Fe}^{\mathrm{II}}(\mathrm{CN})_{6}\right)$ and cubic $(\mathrm{C})$ phase $\left(\mathrm{Fe}^{\mathrm{III}} \mathrm{Fe}^{\mathrm{III}}(\mathrm{CN})_{6}\right) \cdot{ }^{21}$ However, FeHCF NDs/rGO shows a smaller shift during charging/discharging (Fig. 5e, i, Supplementary Fig. 22), indicating a smaller lattice variation as compared with FeHCF MCs/rGO. Moreover, a small unit cell volume variation of below $2.4 \%$ is for 
$246 \mathrm{MCs} / \mathrm{rGO}$ (Fig. 5f, j). The excellent cyclability of FeHCF NDs/rGO can be attributed to the small 247 structural distortion during sodiation/desodiation. The inherent features of ultrafine PB nanocrystals, 248 including a larger lattice, low degree of order, and low structural symmetry, are the most significant 249 contributor to restraining the lattice distortion upon charge/discharge.

250 In summary, we implement the first case of job-sharing storage for the insertion-type cathode materials 251 through the formation of an artificial mixed ion/electron conductor. The job-sharing mechanism, coupled 252 with the size confinement effect, displays several benefits: (1) an additional capacity contribution to 253 compensate for the insertion capacity decrease associated with particle downsizing; (2) the short ion 254 diffusion length and job-sharing transport of ions and electrons lead to outstanding mass transport in the 255 space-charge zone, thereby yielding superb capacities at high C-rates; (3) the lattice expansion and 256 distortion in the size-confined particles limit the structural variation of FeHCF NDs/rGO during $\mathrm{Na}^{+}$ions 257 insertion/extraction, allowing an ultra-stable cyclability. This finding is significant since it is widely 258 applicable to develop insertion-type cathode system of alkali ion secondary batteries. Our work regarding 259 the use of size effect and space-charge effect suggests a new and universal strategy for high-power 260 batteries and also other fields. 
Jung, S.-K. et al. Nanoscale phenomena in lithium-ion batteries. Chem. Rev. 120, 6684-6737 (2020). Maier, J. Pushing nanoionics to the limits: charge carrier chemistry in extremely small systems. Chem. Mater. 26, 348-360 (2014).

Okubo, M. et al. Nanosize effect on high-rate Li-ion intercalation in $\mathrm{LiCoO}_{2}$ electrode. J. Am. Chem. Soc. 129, 7444-7452 (2007).

Perebeinos, V., Chan, S.-W. \& Zhang, F. 'Madelung model' prediction for dependence of lattice parameter on nanocrystal size. Solid State Commun. 123, 295-297 (2002).

Diehm, P. M., Ágoston, P. \& Albe, K. Size-dependent lattice expansion in nanoparticles: reality or anomaly? ChemPhysChem 13, 2443-2454 (2012).

Jamnik, J. \& Maier, J. Nanocrystallinity effects in lithium battery materials apects of nano-ionics. Part IV. Phys. Chem. Chem. Phys. 5, 5215-5220 (2003).

Maier, J. Thermodynamics of electrochemical lithium storage. Angew. Chem. Int. Ed. 52, 4998-5026 (2013).

Qian, D. et al. Electronic spin transition in nanosize stoichiometric lithium cobalt oxide. J. Am. Chem. Soc. 134, 6096-6099 (2012).

Wang, B. et al. $\mathrm{LiFePO}_{4}$ quantum-dots composite synthesized by a general microreactor strategy for ultrahigh-rate lithium ion batteries. Nano Energy 42, 363-372 (2017).

Kobayashi, G. et al. Isolation of solid solution phases in size-controlled $\mathrm{Li}_{\mathrm{x}} \mathrm{FePO}_{4}$ at room temperature. Adv. Funct. Mater. 19, 395-403 (2009).

Okubo, M. et al. Fast Li-ion insertion into nanosized $\mathrm{LiMn}_{2} \mathrm{O}_{4}$ without domain boundaries. ACS Nano 4, 741-752 (2010).

Eum, D. et al. Voltage decay and redox asymmetry mitigation by reversible cation migration in lithiumrich layered oxide electrodes. Nat. Mater. 19, 419-427 (2020).

Lee, J. et al. Reversible $\mathrm{Mn}^{2+} / \mathrm{Mn}^{4+}$ double redox in lithium-excess cathode materials. Nature 556, 185-190 (2018).

Jo, M. R. et al. Triggered reversible phase transformation between layered and spinel structure in manganese-based layered compounds. Nat. Commun. 10, 3385 (2019).

Wang, F. et al. Ternary metal fluorides as high-energy cathodes with low cycling hysteresis. Nat. Commun. 6, 6668 (2015).

Chen, C.-C. \& Maier, J. Decoupling electron and ion storage and the path from interfacial storage to artificial electrodes. Nat. Energy 3, 102-108 (2018).

Chen, C. C. \& Maier, J. Space charge storage in composites: thermodynamics. Phys. Chem. Chem. Phys. 19, 6379-6396 (2017).

Chen, C.-C., Navickas, E., Fleig, J. \& Maier, J. Kinetics of space charge storage in composites. Adv. Funct. Mater. 28, 1705999 (2018).

Chen, C. C., Fu, L. \& Maier, J. Synergistic, ultrafast mass storage and removal in artificial mixed conductors. Nature 536, 159-164 (2016).

Li, W.-J. et al. Facile method to synthesize Na-enriched $\mathrm{Na}_{1+}{ }_{x} \mathrm{FeFe}(\mathrm{CN})_{6}$ frameworks as cathode with superior electrochemical performance for sodium-ion batteries. Chem. Mater. 27, 1997-2003 (2015). You, Y., Wu, X.-L., Yin, Y.-X. \& Guo, Y.-G. High-quality Prussian blue crystals as superior cathode materials for room-temperature sodium-ion batteries. Energy Environ. Sci. 7, 1643-1647 (2014).

Tsunekawa, S. et al. Critical size and anomalous lattice expansion in nanocrystalline $\mathrm{BaTiO}_{3}$ particles. Phys. Rev. B 62, 3065 (2000).

Bhowmik, R., Ranganathan, R. \& Nagarajan, R. Lattice expansion and noncollinear to collinear ferrimagnetic order in a $\mathrm{MnCr}_{2} \mathrm{O}_{4}$ nanoparticle. Phys. Rev. B 73, 144413 (2006). Ali, M. \& Winterer, M. ZnO nanocrystals: surprisingly 'alive'. Chem. Mater. 22, 85-91 (2010). Xie, B. et al. Achieving long-life Prussian blue analogue cathode for Na-ion batteries via triple-cation lattice substitution and coordinated water capture. Nano Energy 61, 201-210 (2019).

Toulouse, J., DiAntonio, P., Vugmeister, B., Wang, X. \& Knauss, L. Precursor effects and ferroelectric macroregions in $\mathrm{KTa}_{1-} \mathrm{Nb}_{\mathrm{x}} \mathrm{O}_{3}$ and $\mathrm{K}_{1-} \mathrm{Li}_{\mathrm{y}} \mathrm{TaO}_{3}$. Phys. Rev. Lett. 68, 232 (1992). 
Lin, T., Cong, X., Lin, M.-L., Liu, X.-L. \& Tan, P.-H. The phonon confinement effect in two-dimensional nanocrystals of black phosphorus with anisotropic phonon dispersions. Nanoscale 10, 8704-8711 (2018). Feng, J. X. et al. Silica-polypyrrole hybrids as high-performance metal-free electrocatalysts for the hydrogen evolution reaction in neutral media. Angew. Chem. 56, 8120-8124 (2017).

29 Wagemaker, M. et al. Dynamic solubility limits in nanosized olivine LiFePO . J. Am. Chem. Soc. 133, 10222-10228 (2011).

30 Tang, Y. et al. Identifying the origin and contribution of surface storage in $\mathrm{TiO}_{2}(\mathrm{~B})$ nanotube electrode by in situ dynamic valence state monitoring. Adv. Mater. 30, 1802200 (2018).

31 Maier, J. Nanoionics: ion transport and electrochemical storage in confined systems. Nat. Mater. 4, 805815 (2005).

32 Fu, L., Chen, C. C., Samuelis, D. \& Maier, J. Thermodynamics of lithium storage at abrupt junctions: modeling and experimental evidence. Phys. Rev. Lett. 112, 208301 (2014).

33 Ren, W. et al. Prussian white hierarchical nanotubes with surface-controlled charge storage for sodium-ion batteries. Adv. Funct. Mater. 29, 1806405 (2019).

34 Huang, Y. et al. A chemical precipitation method preparing hollow-core-shell heterostructures based on the Prussian blue analogs as cathode for sodium-Ion batteries. Small 14, 1801246 (2018).

35 Zhang, W. et al. Synthesis of monocrystalline nanoframes of Prussian blue analogues by controlled preferential etching. Small 55, 8228-8234 (2016).

36 Li, L., Nie, P., Chen, Y. \& Wang, J. Novel acetic acid induced Na-rich Prussian blue nanocubes with iron defects as cathodes for sodium ion batteries. J. Mater. Chem. A 7, 12134-12144 (2019).

37 Huang, Y. et al. A novel border-rich Prussian blue synthetized by inhibitor control as cathode for sodium ion batteries. Nano Energy 39, 273-283 (2017).

$38 \mathrm{Li}$, W. et al. Remarkable enhancement in sodium-Ion kinetics of $\mathrm{NaFe}_{2}(\mathrm{CN})_{6}$ by chemical bonding with graphene. Small Methods 2, 1700346 (2018).

$39 \mathrm{Fu}, \mathrm{H}$. et al. Enhanced storage of sodium ions in Prussian blue cathode material through nickel doping. $J$. Mater. Chem. A 5, 9604-9610 (2017).

$40 \mathrm{Li}, \mathrm{W}$. et al. Stress distortion restraint to boost the sodium ion storage performance of a novel binary hexacyanoferrate. Adv. Energy Mater. 10, 1903006 (2020).

41 Peng, J. et al. A Dual-Insertion type sodium-ion full cell based on high-quality ternary-metal Prussian blue analogs. Adv. Energy Mater. 8, 1702856 (2018).

42 Lesel, B. K., Cook, J. B., Yan, Y., Lin, T. C. \& Tolbert, S. H. Using nanoscale domain size to control charge storage kinetics in pseudocapacitive nanoporous $\mathrm{LiMn}_{2} \mathrm{O}_{4}$ powders. ACS Energy Lett. 2, 2293-2298 (2017).

43 Zhu, C., Usiskin, R. E., Yu, Y. \& Maier, J. The nanoscale circuitry of battery electrodes. Science 358, eaao2808 (2017).

44 Zhang, J. et al. Bifunctional conducting polymer coated CoP core-shell nanowires on carbon paper as a free-standing anode for sodium ion batteries. Adv. Energy Mater. 8, 1800283 (2018).

45 Zhang, J. et al. Engineering solid electrolyte interphase on red phosphorus for long-term and high-capacity sodium storage. Chem. Mater. 32, 448-458 (2020).

46 You, Y. et al. Subzero-temperature cathode for a sodium-Ion battery. Adv. Mater. 28, 7243-7248 (2016). 


\section{Acknowledgements}

355 This work was supported by the National Research Foundation of Korea (NRF) grant funded by the Korea 356 government (MSIP) under "Middle Career Program” (NRF-2017R1A2B3004383), "Science Research 357 Centre" (NRF-2017R1A5A1015365), and "Creative Materials Discovery Program" (NRF358 2017M3D1A1039553).

359 Author contributions

360 Jin.Z. and Y.-M.K. designed the project. Jin.Z. synthesized and characterized the materials and performed 361 the electrochemistry experiments. Jil.Z., G.-H.L. and L.F. performed synchrotron-based measurements 362 and analysed the data. Jin.Z. and M.P. performed TEM, Raman and XPS tests. V.W.L., H.W. and K.Z. 363 offered valuable comments for this project. All authors discussed and analysed the data. Jin.Z., H.W. and 364 Y.-M.K. wrote the manuscript with input from all co-authors.

\section{Competing interests}

366 The authors declare no competing interests.

367 Data availability

368 Any source data are available at https://pan.baidu.com/s/1sjUDZgdLi0jWWor7zbkjZg.

369 Code availability

370 The accession code is publicly available at

371 https://drive.google.com/drive/folders/1nGOcVMJMuWDgBmjOiZIhzvafnmDu8HUy?usp=sharing 372 Additional information 
373 Supplementary information is available for this paper at www.nature.com/nature.

374 Correspondence and requests for materials should be addressed to Y.-M. Kang and H. Wang 375 

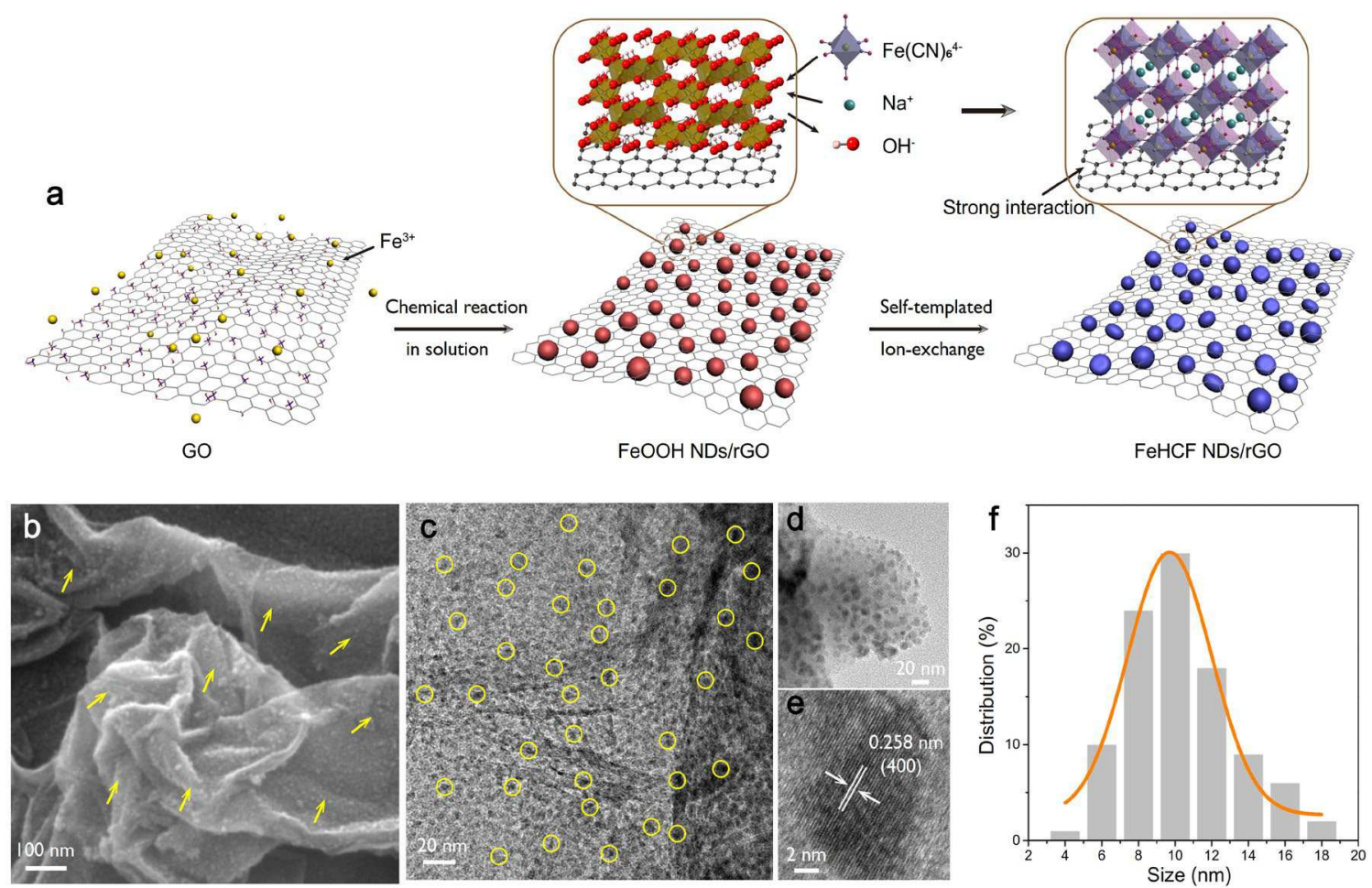

378 Fig. 1 Synthesis process and microstructural analyses of FeHCF NDs/rGO. a, Schematic illustration

379 of the synthesis process by a two-step method. b, SEM image. c, d, TEM images. e) HRTEM image. f, 380 Particle size distribution of FeHCF NDs (200 particles counted). 

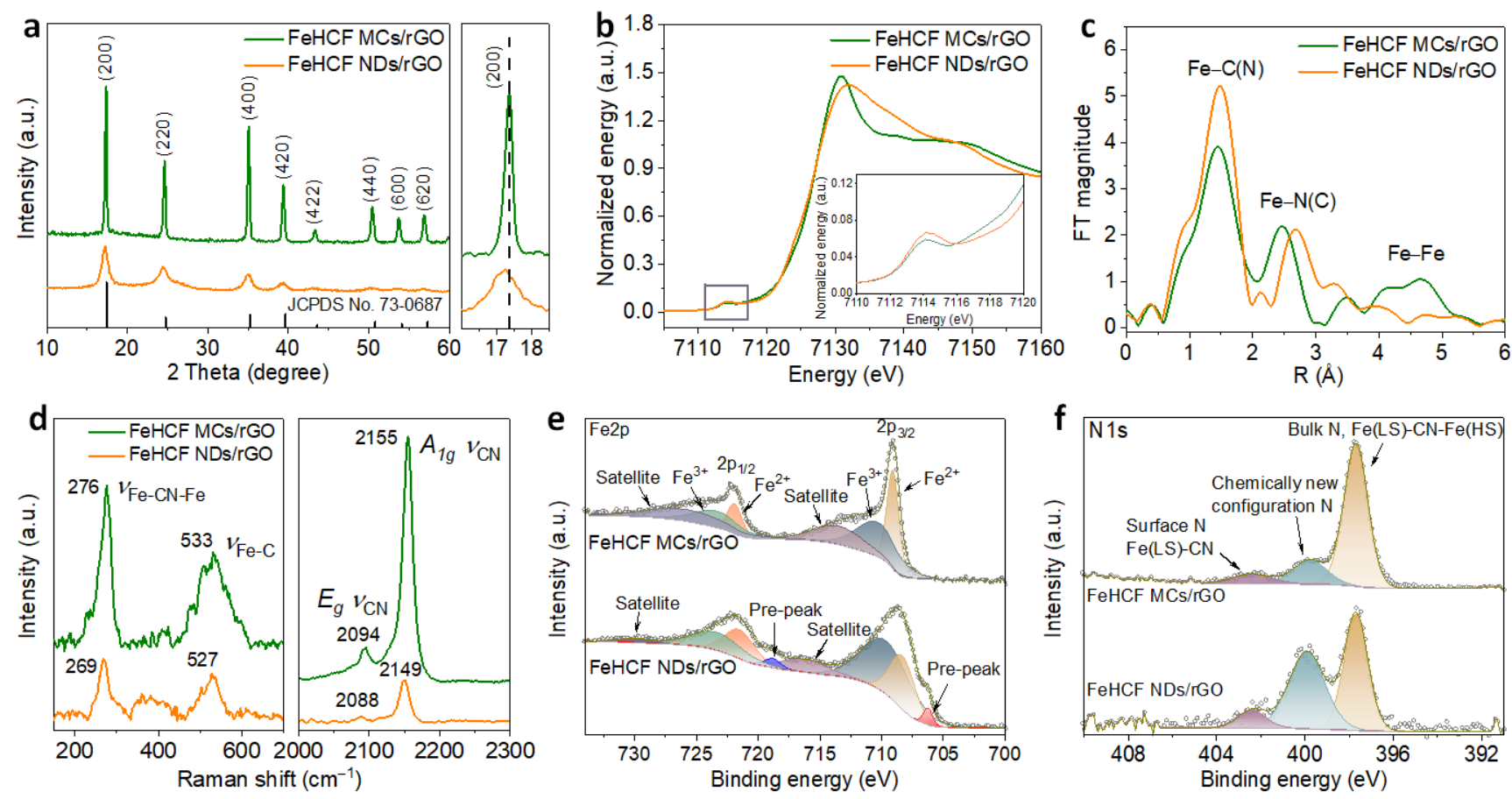

Fig. 2 Structure characterizations of FeHCF MCs/rGO and FeHCF NDs/rGO. a, XRD patterns, 384 including an enlargement of the region around the (200) reflection. b, XANES spectra of the Fe K-edge; 385 inset shows the expanded pre-edge region. c, Fourier transformed EXAFS spectra of the Fe K-edge. d, 386 Raman spectra. e, Fe 2p XPS spectra. f, N 1s XPS spectra. 

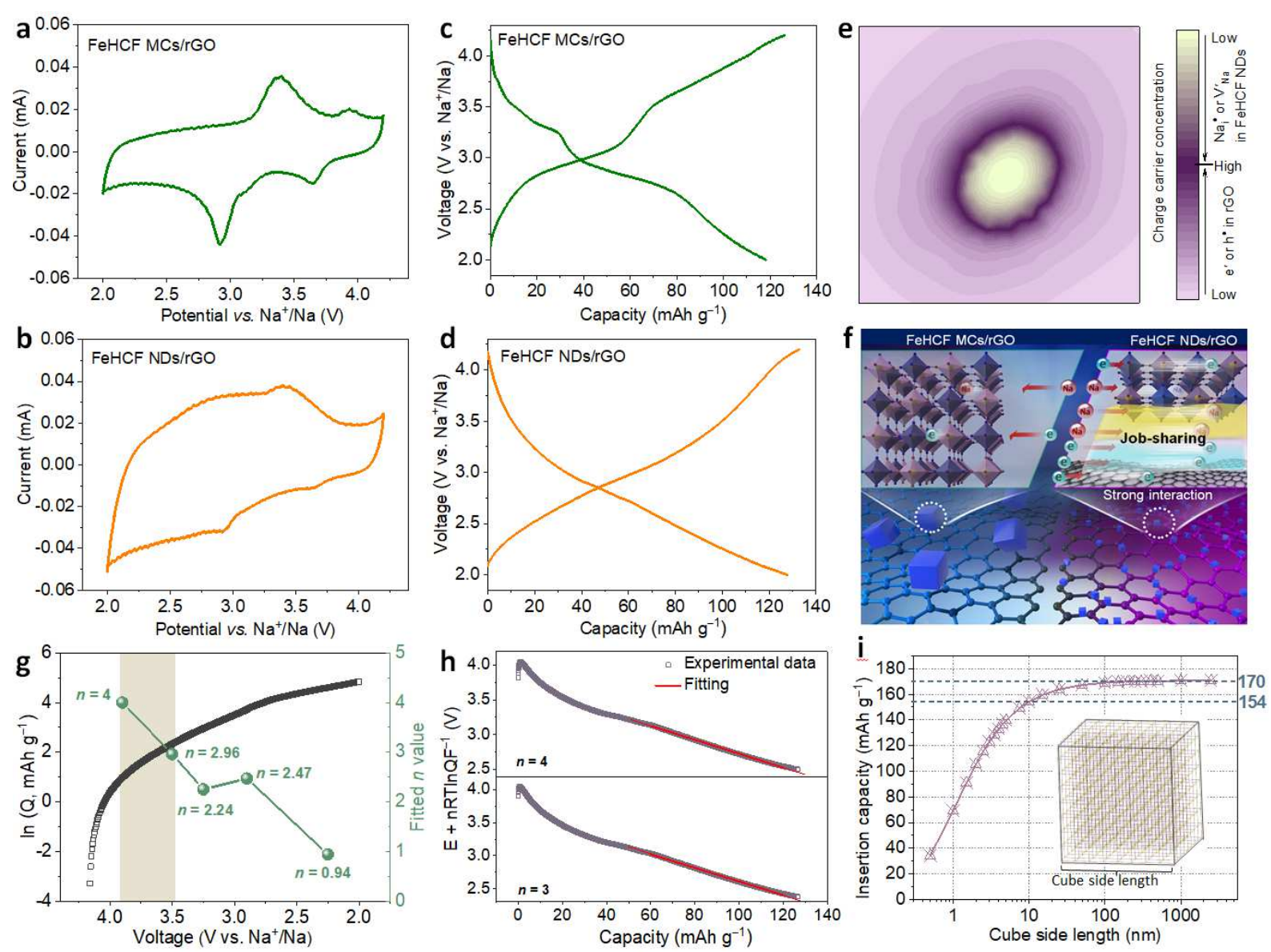

Fig. 3 General electrochemical analyses. Typical CV curves at $0.1 \mathrm{mV} \mathrm{s}^{-1}$ of a FeHCF MCs/rGO and b

FeHCF NDs/rGO. Typical galvanostatic charge/discharge profiles at $0.2 \mathrm{C}\left(1 \mathrm{C}=170 \mathrm{~mA} \mathrm{~g}^{-1}\right)$ of $\mathbf{c}$ FeHCF

MCs/rGO and d FeHCF NDs/rGO. e, Visible redistribution of charge carriers at the FeHCF NDs/rGO interface. $\mathrm{Na}^{+}$interstitials $\mathrm{Na}_{i}{ }^{\circ}$ and $\mathrm{Na}^{+}$vacancies $\mathrm{V}_{\mathrm{Na}}$ are charge carriers in the FeHCF NDs phase, while electron $\mathrm{e}^{\prime}$ and holes $\mathrm{h}^{\circ}$ are charge carriers in the rGO phase. The contour map is derived from Gouy-

Chapman profiles. f, Schematic of and intercalation storage mechanism for FeHCF MCs/rGO and an interfacial storage mechanism for FeHCF NDs/rGO with job-sharing storage and transport of ions and electrons separately. g, Dependence of sodium interfacial storage on sodium activity in FeHCF NDs/rGO nano-composites, where the plot was fitted to the power law function. The power law exponent, $n$, is given at various voltages. $n$ between 3 and 4 is considered to be a reliable fit for charge storage predominantly 
399 in the diffuse layer. h, Dependence of $E+n R T \ln Q / F$ on interfacial storage with $n$ values is 3 and 4 , 400 respectively. The data shows a good linear fitting in high storage. i, Dependence of insertion capacity on 401 the cube length.

402 

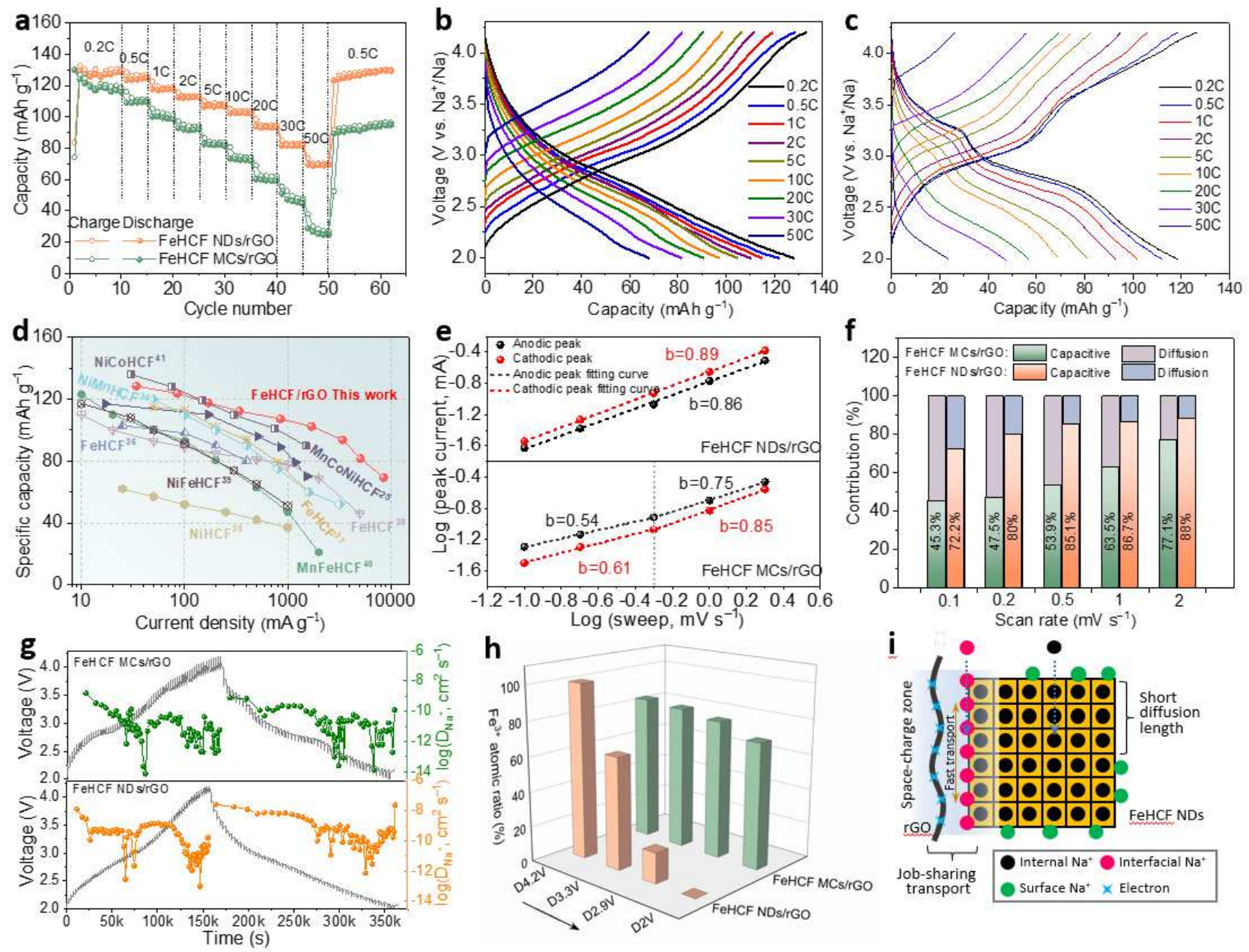

Fig. 4 Reaction kinetics analysis. a, Rate capability of FeHCF NDs/rGO and FeHCF MCs/rGO.

405 Charge/discharge curves at different current densities for $\mathbf{b}$ FeHCF NDs/rGO and $\mathbf{c}$ FeHCF MCs/rGO. d,

406 Comparison of rate capability of other previously reported PBA-based cathode materials. e, Log (peak

407 current) vs. $\log$ (scan rate) plots at cathodic and anodic peaks, and the corresponding linear fitting for the

408 two samples. f, Normalized percentage capacitive contributions of the two samples obtained at different

409 scan rates. g, GITT curves and the calculated $\mathrm{Na}$ ion diffusion coefficients of the two samples. $\mathbf{h}$, The

410 atomic proportion of $\mathrm{Fe}^{\mathrm{III}}$ for the two samples at various discharged states after curve-fitting of XPS

411 spectra. i, Schematic summary of the kinetic mechanism where the interface/surface and the interior of 412 the crystals collaborate effectively. 

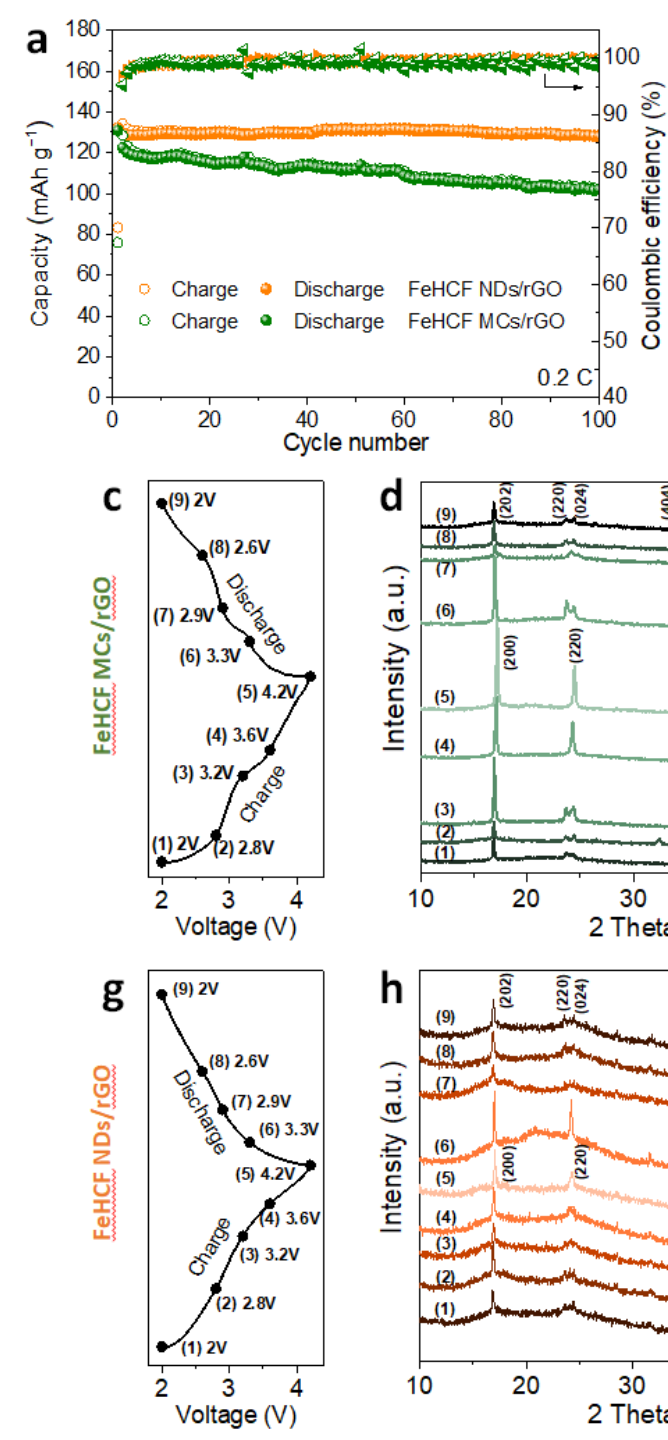
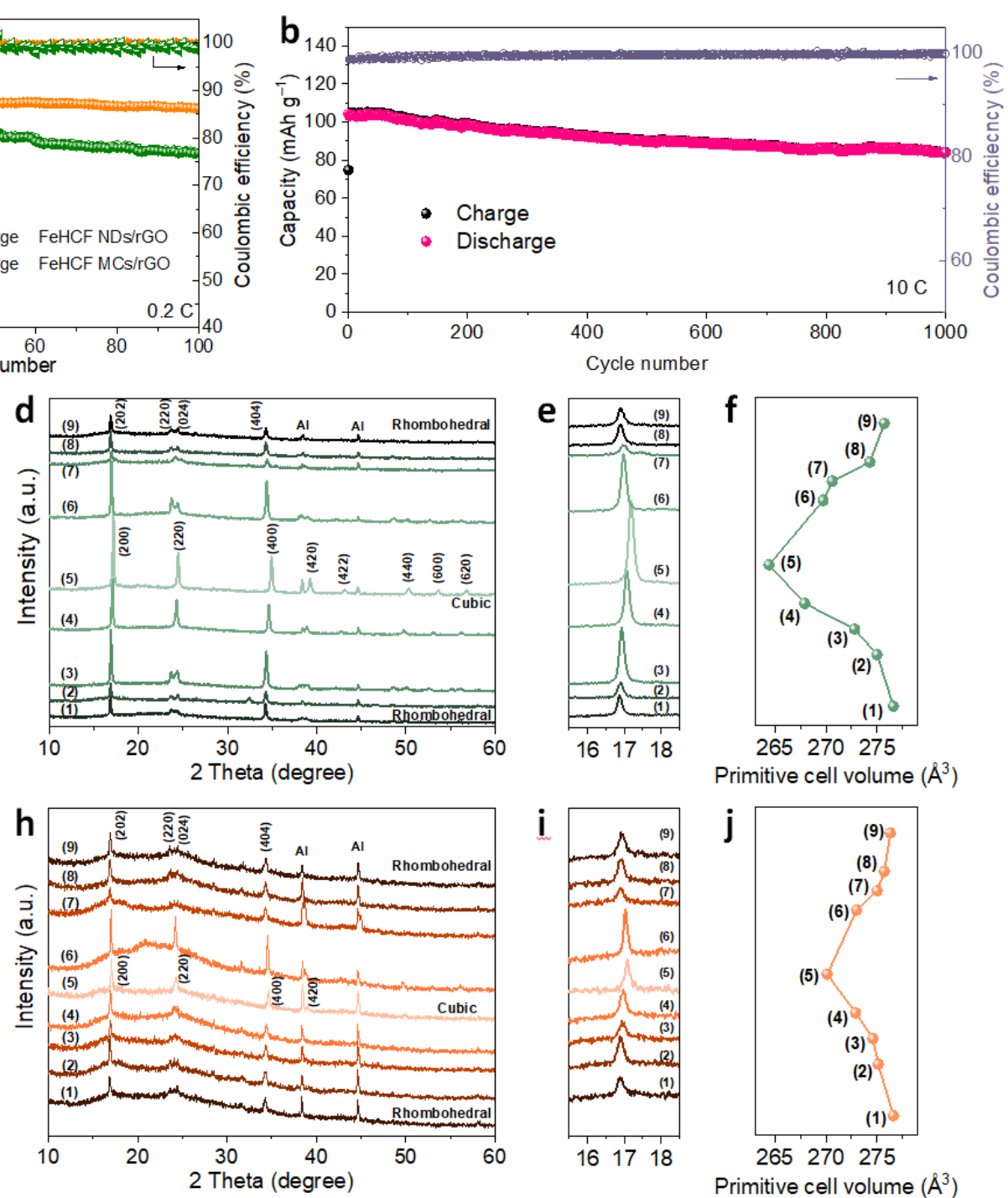

e
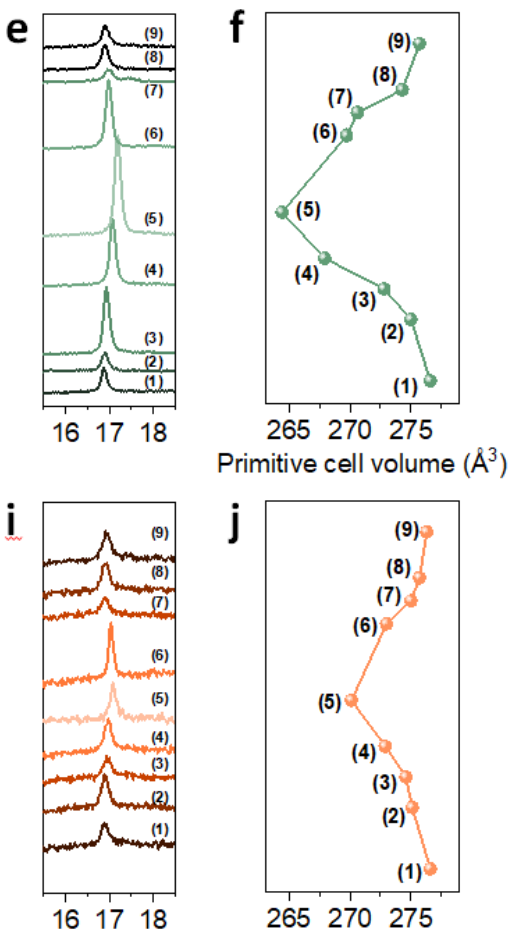

161718

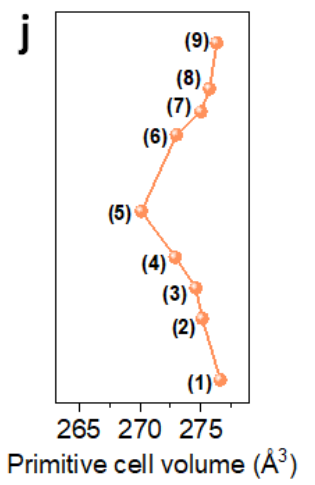

414 Fig. 5 Cyclic stability and structural evolution analyses. a, Cycling performance at $0.2 \mathrm{C} \mathrm{of} \mathrm{FeHCF}$

$415 \mathrm{MCs} / \mathrm{rGO}$ and FeHCF NDs/rGO. b, Long-term cycling performance at $10 \mathrm{C}$ of FeHCF NDs/rGO. Ex-situ 416 characterizations at different states for FeHCF MCs/rGO and FeHCF NDs/rGO: c, g, charge/discharge 417 curves; $\mathbf{d}, \mathbf{h}$, ex-situ XRD patterns; $\mathbf{e}, \mathbf{i}$, enlarged ex-situ XRD patterns; $\mathbf{f}, \mathbf{j}$, corresponding normalized 418 volume variations. 


\section{Figures}
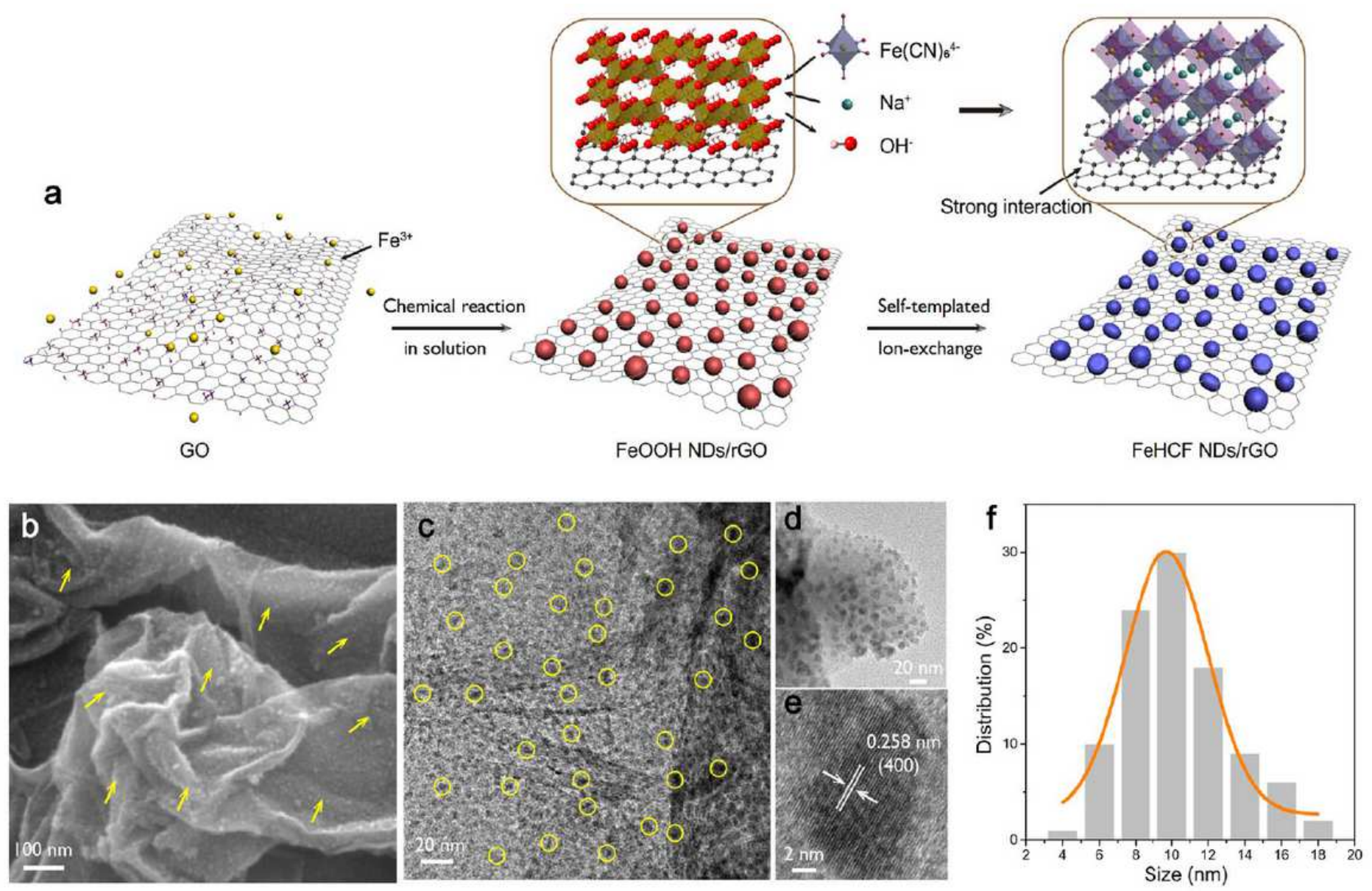

Figure 1

Synthesis process and microstructural analyses of FeHCF NDs/rGO. a, Schematic illustration of the synthesis process by a two-step method. b, SEM image. c, d, TEM images. e) HRTEM image. f, Particle size distribution of FeHCF NDs (200 particles counted). 

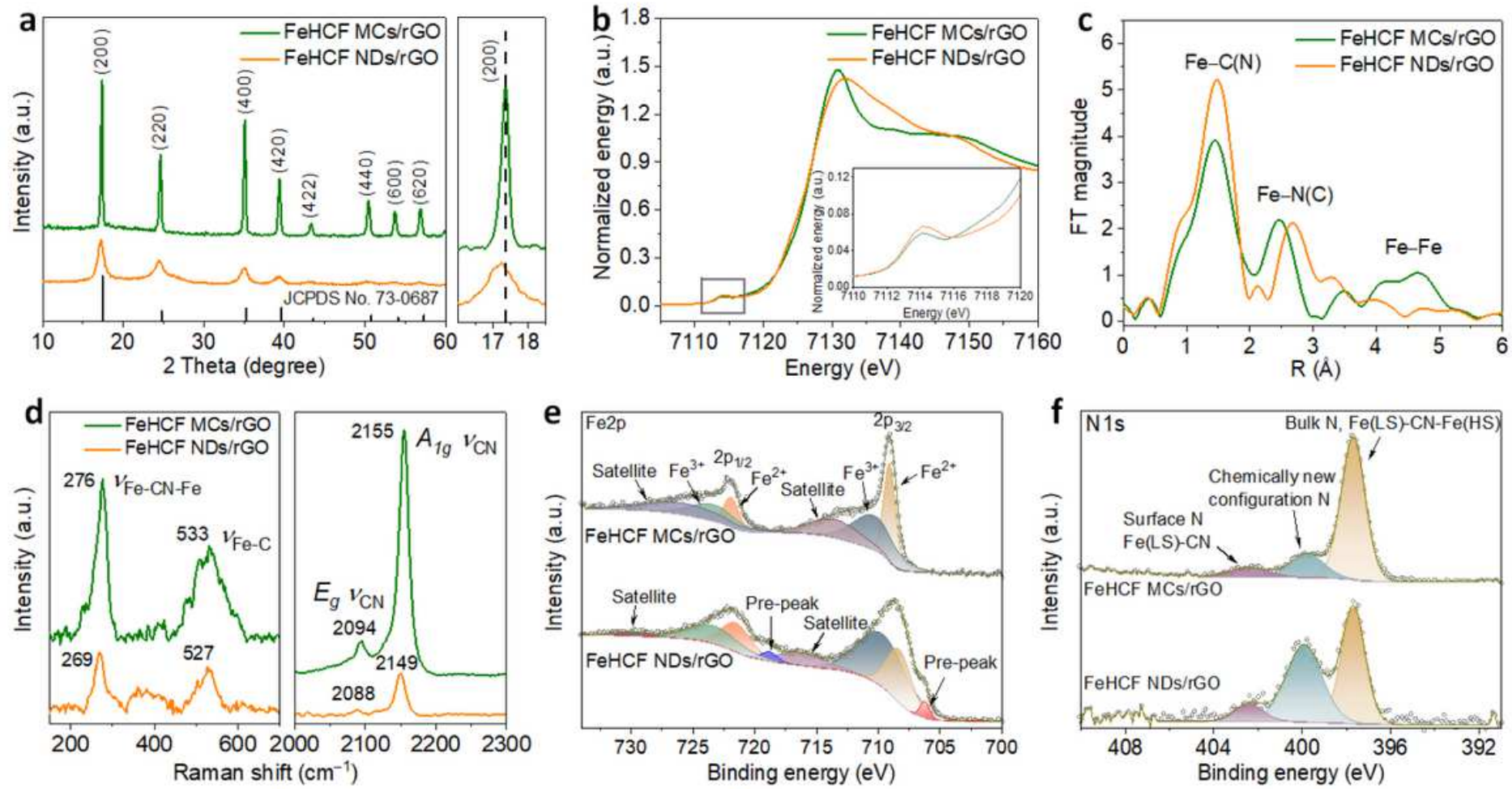

Figure 2

Structure characterizations of FeHCF MCs/rGO and FeHCF NDs/rGO. a, XRD patterns, including an enlargement of the region around the (200) reflection. b, XANES spectra of the Fe K-edge; inset shows the expanded pre-edge region. c, Fourier transformed EXAFS spectra of the Fe K-edge. d, Raman spectra. e, Fe $2 p$ XPS spectra. f, N 1s XPS spectra. 

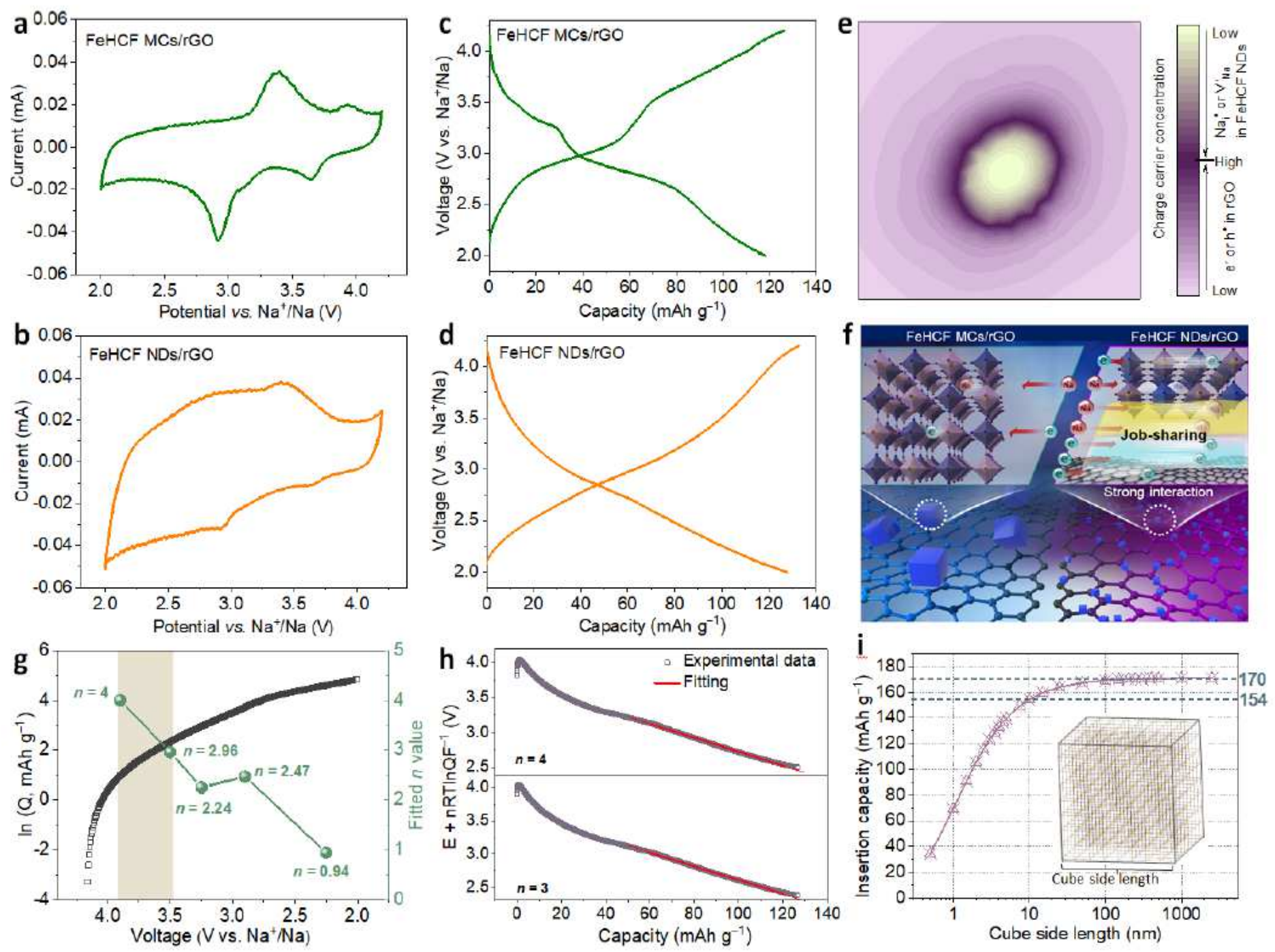

\section{Figure 3}

General electrochemical analyses. Typical CV curves at $0.1 \mathrm{mV} \mathrm{s}-1$ of a FeHCF MCs/rGO and b FeHCF $\mathrm{NDs} / \mathrm{rGO}$. Typical galvanostatic charge/discharge profiles at $0.2 \mathrm{C}(1 \mathrm{C}=170 \mathrm{~mA} \mathrm{~g}-1)$ of c FeHCF $\mathrm{MCs} / \mathrm{rGO}$ and $\mathrm{d}$ FeHCF NDs/rGO. e, Visible redistribution of charge carriers at the FeHCF NDs/rGO interface. $\mathrm{Na}+$ interstitials $\mathrm{Nai}$ and $\mathrm{Na}+$ vacancies VNa' are charge carriers in the FeHCF NDs phase, while electron $e^{\prime}$ and holes $h^{\prime}$ are charge carriers in the rGO phase. The contour map is derived from GouyChapman profiles. $\mathrm{f}$, Schematic of and intercalation storage mechanism for FeHCF MCs/rGO and an interfacial storage mechanism for FeHCF NDs/rGO with job-sharing storage and transport of ions and electrons separately. g, Dependence of sodium interfacial storage on sodium activity in FeHCF NDs/rGO nano-composites, where the plot was fitted to the power law function. The power law exponent, $n$, is given at various voltages. $n$ between 3 and 4 is considered to be a reliable fit for charge storage predominantly in the diffuse layer. h, Dependence of $E+n R T I n Q / F$ on interfacial storage with $n$ values is 3 and 4 , respectively. The data shows a good linear fitting in high storage. i, Dependence of insertion capacity on the cube length. 

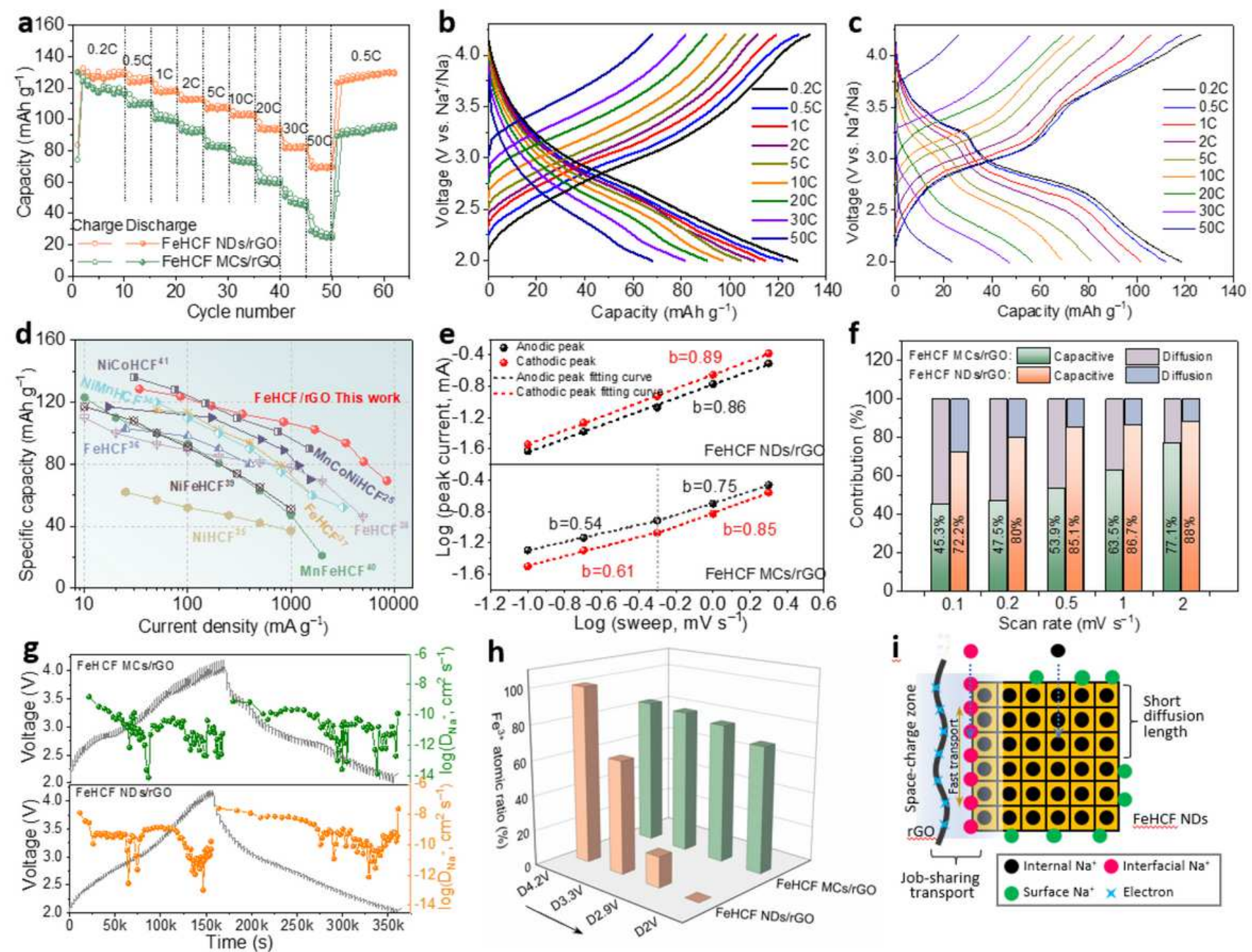

\section{Figure 4}

Reaction kinetics analysis. a, Rate capability of FeHCF NDs/rGO and FeHCF MCs/rGO. Charge/discharge curves at different current densities for b FeHCF NDs/rGO and c FeHCF MCs/rGO. d, Comparison of rate capability of other previously reported PBA-based cathode materials. e, Log (peak current) vs. log (scan rate) plots at cathodic and anodic peaks, and the corresponding linear fitting for the two samples. $f$, Normalized percentage capacitive contributions of the two samples obtained at different scan rates. $\mathrm{g}$, GITT curves and the calculated Na ion diffusion coefficients of the two samples. h, The atomic proportion of Fe区 for the two samples at various discharged states after curve-fitting of XPS spectra. i, Schematic summary of the kinetic mechanism where the interface/surface and the interior of the crystals collaborate effectively. 

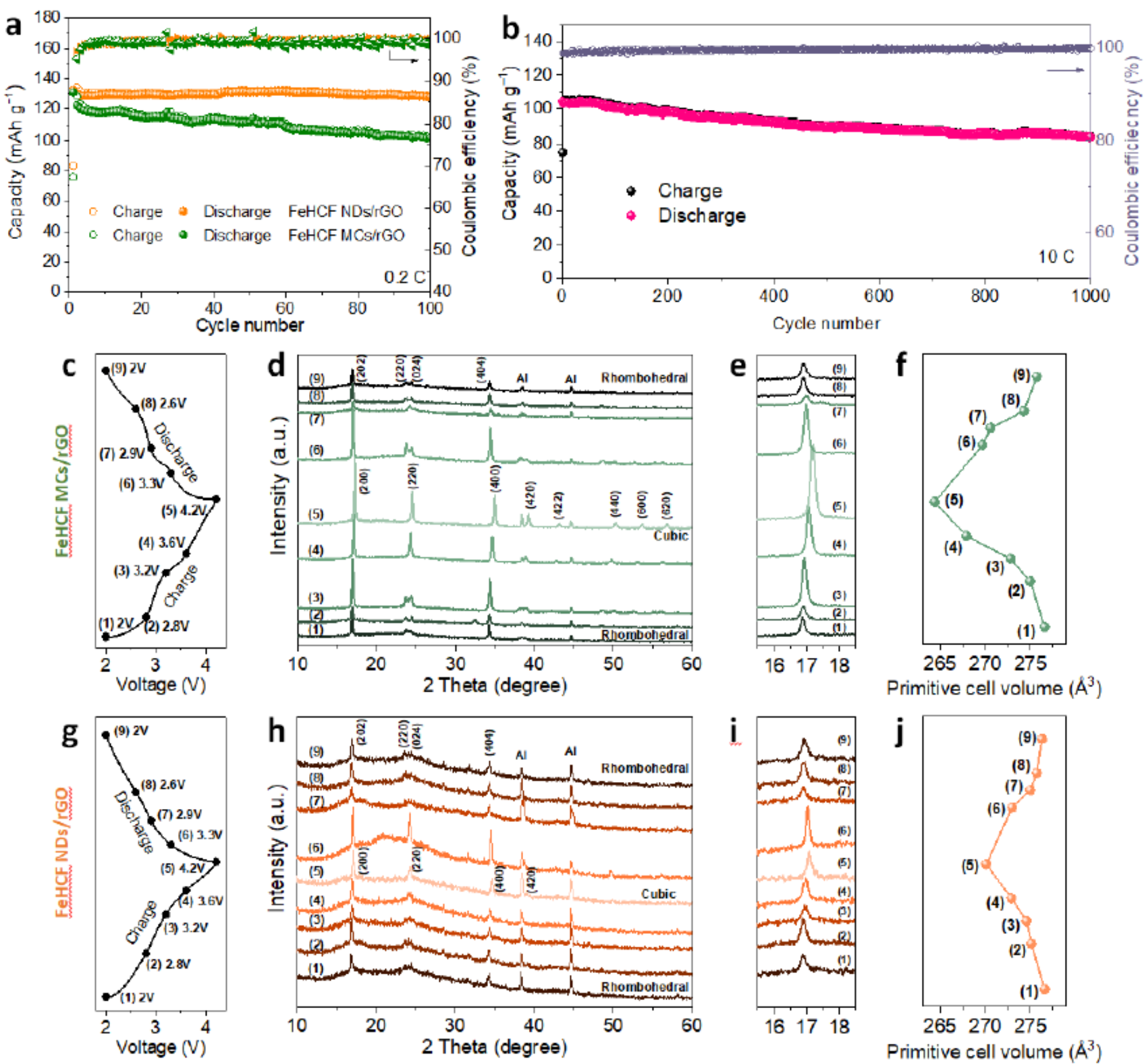

265270275

Primitive cell volume $\left(\AA^{3}\right)$

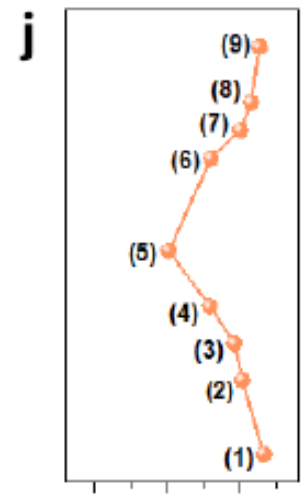

265270275

Primitive cell volume $\left(\AA^{3}\right)$

\section{Figure 5}

Cyclic stability and structural evolution analyses. a, Cycling performance at $0.2 \mathrm{C}$ of FeHCF MCs/rGO and FeHCF NDs/rGO. b, Long-term cycling performance at $10 \mathrm{C}$ of FeHCF NDs/rGO. Ex-situ characterizations at different states for FeHCF MCs/rGO and FeHCF NDs/rGO: c, g, charge/discharge curves; d, h, ex-situ XRD patterns; e, i, enlarged ex-situ XRD patterns; f, j, corresponding normalized volume variations.

\section{Supplementary Files}

This is a list of supplementary files associated with this preprint. Click to download. 
- SupportinginformationNatureYMKHHWFinal1.pdf 